Article

\title{
Identification of Site-Specific Stroke Biomarker Candidates by Laser Capture Microdissection and Labeled Reference Peptide
}

\author{
Tingting Lian ${ }^{\dagger}$, Daixin $\mathbf{Q u}^{\dagger}$, Xu Zhao, Lixia Yu and Bing Gao * \\ School of Bioscience and Bioengineering, South China University of Technology, \\ Higher Education Mega Center, Guangzhou 510006, China; E-Mails: l.tt04@mail.scut.edu.cn (T.L.); \\ qu.daixin@mail.scut.edu.cn (D.Q.); rogerxu8911@gmail.com (X.Z.); yulixia2015@gmail.com (L.Y.) \\ † These authors contributed equally to this work.
}

* Author to whom correspondence should be addressed; E-Mail: binggao@scut.edu.cn; Tel./Fax: +86-20-3938-0618.

Academic Editor: David Sheehan

Received: 4 April 2015 / Accepted: 3 June 2015 / Published: 11 June 2015

\begin{abstract}
The search to date for accurate protein biomarkers in acute ischemic stroke has taken into consideration the stage and/or the size of infarction, but has not accounted for the site of stroke. In the present study, multiple reaction monitoring using labeled reference peptide (LRP) following laser capture microdissection (LCM) is used to identify site-specific protein biomarker candidates. In middle cerebral artery occlusion (MCAO) rat models, both intact and infarcted brain tissue was collected by LCM, followed by on-film digestion and semi-quantification using triple-quadrupole mass spectrometry. Thirty-four unique peptides were detected for the verification of 12 proteins in both tissue homogenates and LCM-captured samples. Six insoluble proteins, including neurofilament light polypeptide (NEFL), alpha-internexin (INA), microtubule-associated protein 2 (MAP2), myelin basic protein (MBP), myelin proteolipid protein (PLP) and 2',3'-cyclic-nucleotide 3'-phosphodiesterase (CNP), were found to be site-specific. Soluble proteins, such as neuron-specific enolase (NSE) and ubiquitin carboxyl-terminal hydrolase isozyme L1 (UCHL1), and some insoluble proteins, including neurofilament heavy polypeptide (NEFH), glial fibrillary acidic protein (GFAP), microtubule-associated protein tau (MAPT) and tubulin $\beta$-3 chain (TUBB3), were found to be evenly distributed in the brain. Therefore, we conclude that some insoluble protein biomarkers for stroke are site-specific, and would make excellent candidates for the design and analysis of relevant clinical studies in the future.
\end{abstract}


Keywords: laser capture microdissection (LCM); labeled reference peptide (LRP); blood-brain barrier (BBB); stroke; biomarker; multiple reaction monitoring (MRM)

\section{Introduction}

Numerous studies have focused on blood biomarker discovery for ischemic stroke and a variety of protein biomarker candidates have been identified [1]. However, the accuracy and reproducibility of these biomarker candidates still need to be improved. The release of glial and neuronal proteins into the blood varies according to the complexity and heterogeneity of lesions after a stroke. The plasmatic levels of protein biomarkers have been correlated to age, genetic background, gender and predisposing factors, such as infection and inflammation, as well as the stage and/or the size of infarction [2-5]. However, the site of infarction has not been considered as an important indicator in previous clinical studies [1,6], and more effort is required to identify site-specific protein biomarker candidates for acute ischemic stroke.

Accurate measurement of protein levels in infarcted tissue is a challenge because the weight of tissue sample cannot be used for normalization due to its heterogeneous composition. Laser capture microdissection (LCM) provides an approach to make normalization possible based on the area of tissue sample, and thus it could become a new assay to assess protein distribution and stability in infarcted brain tissues. Since 1996, traditional tissue cutting has been replaced by LCM for procuring pure cell populations in a quick and automated manner [7]. In 2014, Subramanian et al. collected the fluorescently labeled neurons from sections encompassing the rostrocaudal extent of the rostral ventrolateral portion of the medulla by LCM [8]. In 2014, genetically labeled neurons from the drosophila melanogaster brain were isolated by LCM, and the catapulted tissue patches were collected on a reversed phase column, and analyzed using an on-column extraction (OCE) that was directly coupled with liquid chromatography-multistage mass spectrometry (LC-MS(n)) [9].

Targeted proteins from LCM-dissected homogeneous cell populations can be quantitatively analyzed by multiple reaction monitoring (MRM) using stable isotope dilution (SID). Bagnato et al., measured cytokines and growth factors in coronary arteries by MRM assay. Four ${ }^{13} \mathrm{C} /{ }^{15} \mathrm{~N}$-labeled peptides for cytokines/growth factors were spiked into LCM-procured samples to complement the shotgun proteomics approaches for low abundance protein identification [10]. In 2011, Guzel et al., measured preeclampsia-related calcyclin peptides using peptides with an extra glycine inserted in the endogenous sequence as an internal standard instead of the heavy labeling method for MRM assay [11]. Attempts have been made to quantify proteins of interest at a low cost. In 2011, Zhang et al. developed a labeled reference peptide (LRP) method that uses a single labeled peptide as a reference standard for all targeted peptides and measures peptides based on their un-normalized peak areas for detected MRM transitions [12].

As indicated above, LCM followed by MRM using LRP (LCM-LRP) is an established tool for screening site-specific protein biomarkers in both intact and infarcted tissue. By using LCM-LRP, we can normalize the level of targeted protein by the capture area of LCM that remains the same for infarcted tissue and intact tissue. The procedure used in the present study is summarized in Figure 1A. 
We procured brain tissue samples from middle cerebral artery occlusion (MCAO) rat model brain by LCM and measured proteins of interest by triple-quadrupole mass spectrometry using the LRP method. Samples included infarcted brain tissue from zones 1-3 and intact brain tissue from zones 4-6 (Figure 1D). Thirty-four unique peptides of 12 proteins are verified and semi-quantified in both brain tissue homogenates and LCM-procured samples. Reference peptides, with or without ${ }^{13} \mathrm{C}$ and ${ }^{15} \mathrm{~N}$ labeling, and maltose-binding protein (mbp) are added to all samples for quality control purposes. Peak area ratios of each unique peptide in zones 1-3 and 4-6 were measured and compared, the distribution of the corresponding protein evaluated, and site-specific protein biomarkers identified.

A

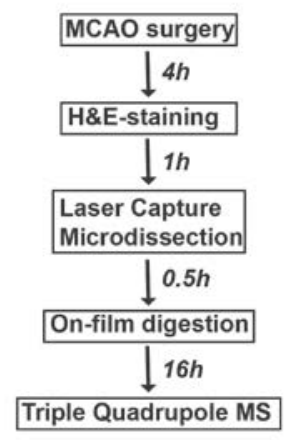

C

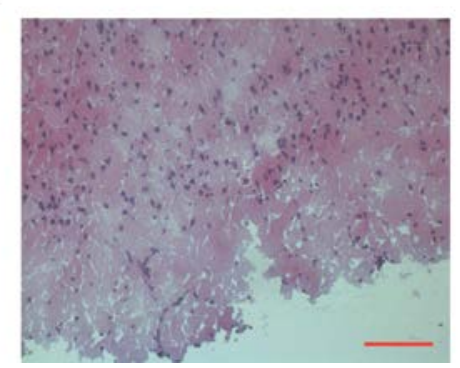

B

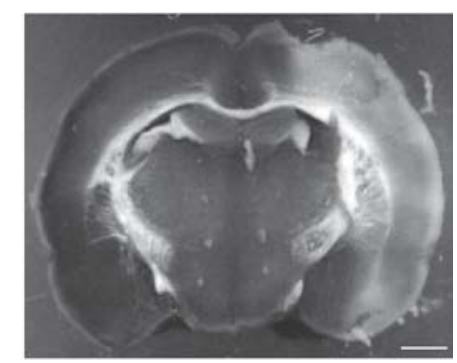

D

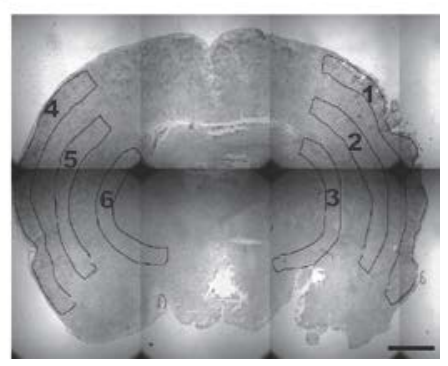

Figure 1. The focal cerebral ischemic model. (A) Scheme of sample collection, processing and analysis; (B) The extent of cerebral infarct was shown by TTC (2,3,5-triphenyltetrazolium chloride) staining. Scale bar: $1 \mathrm{~mm}$; (C) Representative hematoxylin and eosin (H\&E)-stained mouse brain sections at $4 \mathrm{~h}$ after the occlusion of middle cerebral artery. Scale bar: $100 \mu \mathrm{m}$; (D) Laser capture microdissection (LCM) of intact and infarcted tissue in the cerebral cortex. Microscopic findings of focal ischemic tissue in the right cerebral cortex and normal tissue in the left cerebral cortex. Coronally cut fresh sections were stained with $\mathrm{H} \& \mathrm{E}$ and shown at a low magnification. Zones 1-3 of ischemic brain tissue supplied by right middle cerebral artery were collected by LCM. The corresponding areas (zones 4-6) in the left cerebral cortex were also collected as a control. The size of each area was measured. Scale bar: $1 \mathrm{~mm}$.

\section{Results and Discussion}

\subsection{The Focal Cerebral Ischemic Model in Rats}

Most human ischemic strokes are caused by occlusion of the middle cerebral artery (MCA), and thus intraluminal MCA occlusion model in rat is the most common model of focal cerebral ischemia. 
In the present study, we established a permanent focal cerebral ischemia model by inserting a monofilament into the internal carotid artery (ICA) to block blood flow to the MCA for $4 \mathrm{~h}$, and then grade neurological deficits of these MCAO animals by Longa neurologic score [13]. All MCAO rats in this study are scored 2.0 (circulating to the left) and considered as successful focal cerebral ischemic models (supplementary video 1 ).

The area of infarction after occlusion of the MCA is identified by 2,3,5-triphenyltetrazolium chloride (TTC) staining, which makes infarcted regions white (Figure 1B). Size and location of the infarction were determined by hematoxylin and eosin (H\&E) staining (Figure 1C). Condensed, dead neurons with eosinophilic cytoplasm and/or pyknosis are scattered throughout the spongy edematous necrotic neutrophil. The swollen astrocytic processes are arranged in parallel. There is evidence of infiltration by monocytes and macrophages, as well as their mitotic figures.

\subsection{Laser Capture Microdissection (LCM) of Intact and Infarcted Tissue in the Cerebral Cortex}

To quantitatively analyze protein levels in the infarct region, both intact and infarcted tissue on the same brain slice were collected by LCM at a low magnification (Figures 1D and 2). Coronally cut fresh sections were stained with $\mathrm{H} \& \mathrm{E}$ and focal infarct areas in the right cerebral cortex were identified. Based on both TTC and H\&E staining, the cerebral cortex was divided into three zones, with the outer layer designated as zone 1 , the inner layer as zone 3 , and the middle layer as zone 2 . These zones are supplied by the right middle cerebral artery before occlusion. The corresponding zones (4-6) in the left cerebral cortex were also collected as a control. The total captured area of each zone was $10 \mathrm{~mm}^{2}$ (Table $1, n=12$ ).

A

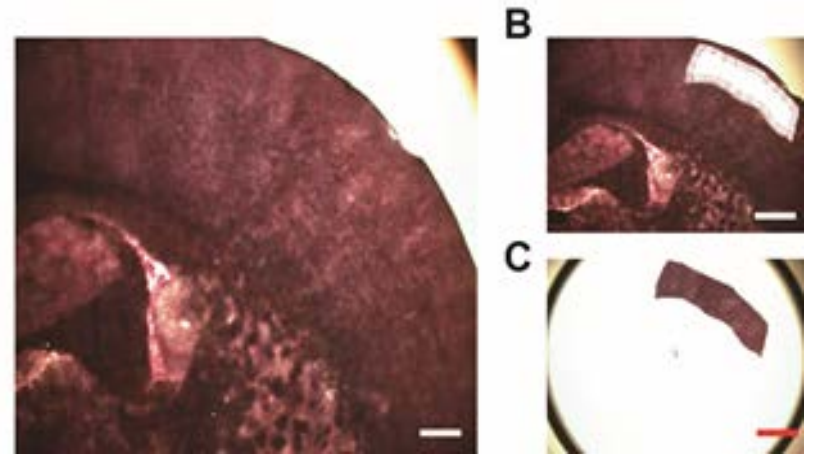

Figure 2. Sample collection by laser capture microdissection (LCM). (A) Infarcted tissue in the cerebral cortex before LCM. Scale bar: $0.5 \mathrm{~mm}$; (B) Infarcted tissue in the cerebral cortex after LCM. Scale bar: $1.0 \mathrm{~mm}$; (C) LCM-procured tissue samples. Scale bar: $1.0 \mathrm{~mm}$.

Table 1. Area of brain tissue obtained by laser capture microdissection (LCM).

\begin{tabular}{ccccccc}
\hline Sample Size & Zone 1 & Zone 2 & Zone 3 & Zone 4 & Zone 5 & Zone 6 \\
\hline LCM Area $\left(\mathrm{mm}^{2}\right)$ & $10.36 \pm 0.07$ & $9.96 \pm 0.08$ & $9.67 \pm 0.05$ & $10.47 \pm 0.07$ & $9.87 \pm 0.07$ & $9.71 \pm 0.07$ \\
\hline
\end{tabular}

2.3. Semi-Quantification of Quality Control Protein by Labeled Reference Peptide (LRP) Analysis

One ng of the unique peptide (APGLTQALNTK*) with ${ }^{13} \mathrm{C}$ and ${ }^{15} \mathrm{~N}$ labeling at the C-terminal lysine and $1 \mathrm{ng}$ of the same peptide without labeling were added to all samples as reference peptides 
(RP). The peak area of unlabeled RP is 25,237 \pm 766.7 (counts) and that of labeled RP (RP*) is $25,952 \pm 815.9$ (Figure 3A, $n=72$ ). The peak area ratio of RP versus $\mathrm{RP}^{*}$ is $0.9959 \pm 0.02496$ (Table 2, $n=72$ ), as compared to a theoretical value of 1.0000 .

Table 2. Quantification of unlabeled reference peptide (RP), labeled reference peptide $(\mathrm{RP} *)$ and maltose-binding protein (mbp) unique peptides.

\begin{tabular}{cccccc}
\hline Peptide & Sequence & $\begin{array}{c}\text { Molecular } \\
\text { Weight (Da) }\end{array}$ & Peak Area & $\begin{array}{c}\text { Peak Area Ratio } \\
\text { (RP*) }\end{array}$ & $\begin{array}{c}\text { Theoretical } \\
\text { Amount (ng) }\end{array}$ \\
\hline RP & APGLTQALNTK & 1113.261 & $25,237 \pm 766.7$ & $0.9959 \pm 0.02496$ & 1 \\
RP* & APGLTQALNTK* & 1121.261 & $25,952 \pm 815.9$ & 1.0000 & 1 \\
mbp-Pa & DVGVDNAGAK & 944.979 & $77,202 \pm 5126$ & $3.182 \pm 0.1818$ & 3.336641 \\
mbp-Pb & AGLTFLVDLIK & 1189.442 & $65,117 \pm 3118$ & $2.687 \pm 0.1039$ & 4.199819 \\
mbp-Pc & VNYGVTVLPTFK & 1337.562 & $103,733 \pm 5828$ & $4.242 \pm 0.1669$ & 4.722818 \\
\hline
\end{tabular}

A total of $150 \mathrm{ng}$ of maltose-binding protein (mbp, M.W. 42,481.902 Da) was added to all samples and the peak areas of three mbp unique peptides (mbp-Pa: DVGVDNAGAK, mbp-Pb: AGLTFLVDLIK, mbp-Pc: VNYGVTVLPTFK) were measured (Table 2). The peak area ratio of each mbp unique peptide (UP) versus $\mathrm{RP}^{*}(\mathrm{UP} / \mathrm{RP} *$ ) indicates the amount of that unique peptide estimated by LRP analysis. These values and the corresponding theoretical amounts for each mbp peptide, equal to $150 \mathrm{ng} \times(\mathrm{M}$.W. of mbp peptide/M.W. of MBP), are shown in Table 2. For example, the estimated amount of mbp-Pa is $3.182 \pm 0.1818 \mathrm{ng}$, a value close to its theoretical amount of $3.336641 \mathrm{ng}$. The standard errors of peak area ratios of mbp- $\mathrm{Pa},-\mathrm{Pb}$ and $-\mathrm{Pc}$ are all small enough to allow a reliable semi-quantitative analysis of mbp protein (Table 2, Figure 3B, $n=72$ ).

A

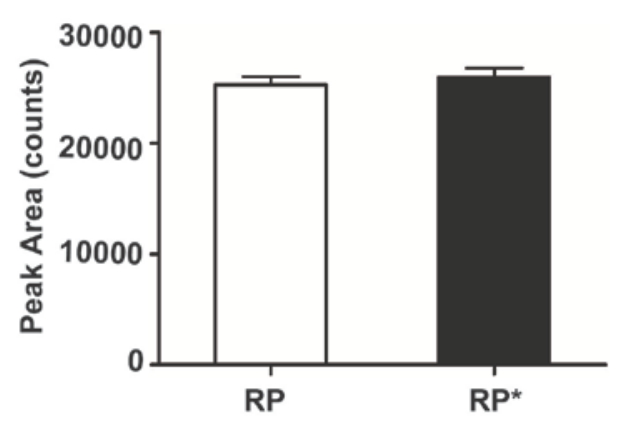

B

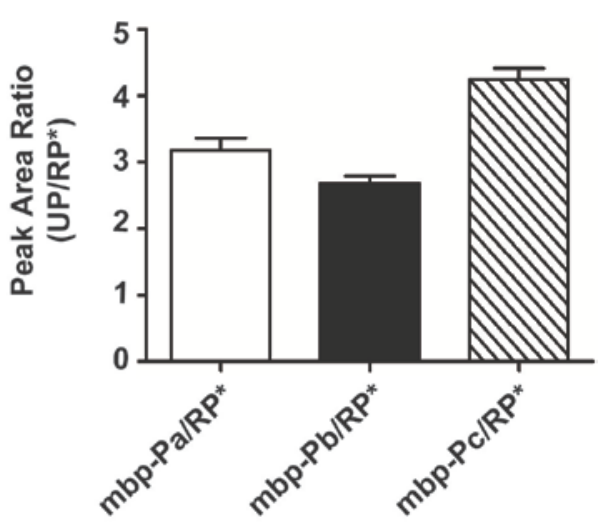

Figure 3. Semi-quantitative analysis of LCM-procured samples by triple-quadrupole mass spectrometry. (A) One ng of ${ }^{13} \mathrm{C}$ and ${ }^{15} \mathrm{~N}$ labeled reference peptide (APGLTQALNTK*) and $1 \mathrm{ng}$ of unlabeled reference peptide (APGLTQALNTK) were added to all samples as a quality control ( $n=72$ ); (B) A total of $150 \mathrm{ng}$ of maltose-binding protein (mbp) was added to all samples and the peak areas of three mbp unique peptides (mbp-Pa, DVGVDNAGAK; mbp-Pb, AGLTFLVDLIK; mbp-Pc, VNYGVTVLPTFK) were measured ( $n=72)$. The peak area ratio of each mbp unique peptide to $\mathrm{RP}^{*}$ is shown. $\mathrm{RP}$, unlabeled reference peptide; $\mathrm{RP} *$, labeled reference peptide. 


\subsection{Distribution of Neurofilament- and Microtubule-Related Proteins in both Intact and Infarcted} Brain Tissues

After focal ischemia, blood-brain barrier (BBB) permeability increases at the site of infarction or the distant region of the ipsilateral cerebrum, allowing blood proteins such as albumin to cross the $\mathrm{BBB}$ and enter the interstitial space, while glial and neuronal proteins enter the blood [14]. Biomarker protein candidates, including glial fibrillary acidic protein (GFAP), myelin basic protein (MBP) and neuron-specific enolase (NSE) are released into the blood through the disrupted blood-brain barrier. In this study, LCM-captured samples from zones 4-6 in intact brain tissues and zones 1-3 in infarcted brain tissues were analyzed by LRP using a triple-quadrupole mass spectrometer. Four neurofilament proteins, including neurofilament light polypeptide (NEFL), neurofilament heavy polypeptide (NEFH), alpha-internexin (INA) and glial fibrillary acidic protein (GFAP), and three microtubule proteins, including microtubule-associated protein 2 (MAP2), microtubule-associated protein tau (MAPT) and tubulin beta-3 chain (TUBB3), were verified by their unique peptides (Table S1).

Unique peptides with the greatest peak area for each protein are highlighted in Table S1 and used for semi-quantification of their corresponding proteins. GFAP is a monomeric intermediate filament protein in astrocytes and its protein level in the serum reaches a maximum between day 2 and 4 after acute ischemic stroke [15]. Serum GFAP is detectable within $6 \mathrm{~h}$ after acute intracerebral hemorrhage (ICH) with a sensitivity of 0.79 and a specificity of 0.98 [16]. By LCM-LRP, we found that GFAP is evenly distributed in zones 4-6 or zones 1-3 (Figure 4A,B), which suggests that the plasma level of GFAP is not related to the site of stroke. NEFH is a major component of the intermediate filaments in neurons, and it plays an important role in mature axons [17]. Our results indicate that NEFH is also evenly distributed in both intact and infarcted brain tissues (Figure 4A,B), and thus not site-specific. INA is the counterpart of GFAP in neurons and it may form an intermediate filament network with or without the involvement of NEFL [18]. INA is believed to play a role in axonal outgrowth. Lower levels of INA and NEFL were found in zone 4 than zones 5-6, which indicates that more INA and NEFL is present in the inner layer of cerebral cortex. MAPs and tubulin are major components of microtubules, with MAP2 being found mostly in dendrites and MAPT in the axon [19,20]. In the present study, more MAP2 was found in zone 4 than zones 5 and 6, but MAPT and TUBB3 were evenly distributed in all areas of intact brain tissues (Figure 4C, Table S2, $n=12$ ), and the distribution pattern of these biomarker candidates was similar in zones 1-3 (Figure 4D, Table S2, $n=12$ ).

A

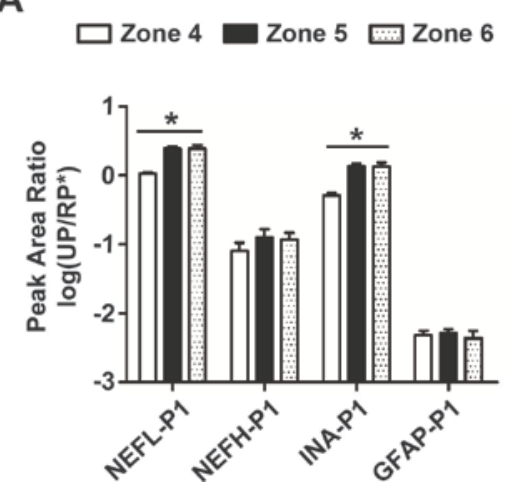

B

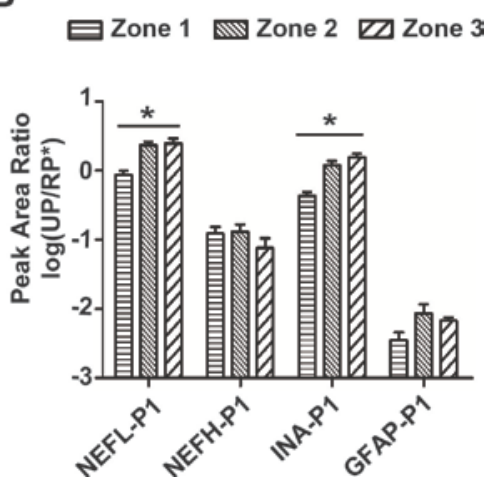

Figure 4. Cont. 
C

$\square$ Zone $4 \square$ Zone 5 Wone 6

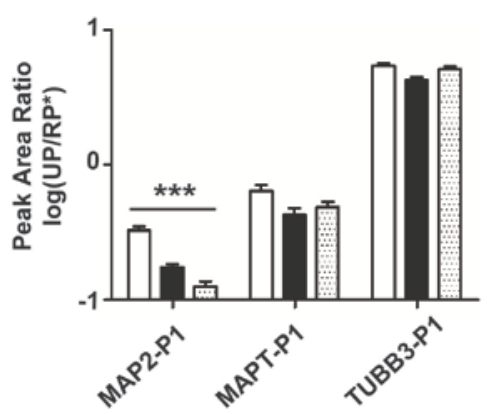

鸟Zone 1 Zone 2 Zone 3

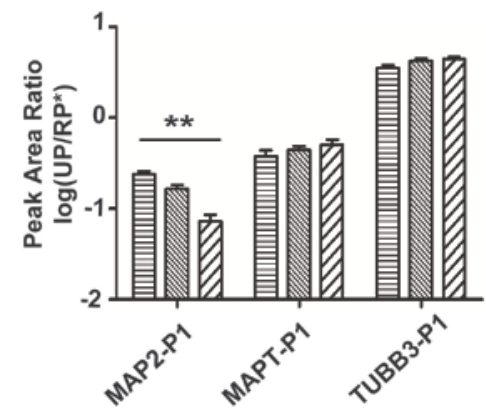

Figure 4. Distribution of neurofilament and microtubule peptides/proteins in both intact and infarcted brain tissues. (A) Proteins in zones 4-6 of intact brain tissue were collected by LCM, $10 \mathrm{~mm}^{2}$ for each area, and then analyzed by a triple-quadrupole mass spectrometer. Four neurofilament proteins were verified and semi-quantified by LCM-LRP. Each protein was verified by $2-3$ unique peptides and quantified by the peak area. The unique peptides with the greatest peak area are shown. Peak area ratios of unique peptides to RP* were calculated (UP/RP*) $(n=12)$; (B) Four neurofilament proteins in zones $1-3$ of infarcted brain tissue were collected by LCM, and then the peak area ratios of 4 unique peptides to RP* were determined ( $n=12$ ); (C) There were 3 microtubule proteins from zones 4-6 of intact brain tissues verified and semi-quantified by LCM-LRP. Each protein was verified by 2-3 unique peptides and quantified by the peak area. The unique peptides with the greatest peak area are shown. Peak area ratios of unique peptides to RP* were calculated (UP/RP*) ( $n=12)$; (D) Three microtubule proteins in zones 1-3 of infarcted brain tissue are collected by LCM, and then the peak area ratios of 3 unique peptides to $\mathrm{RP}^{*}$ were determined $(n=12)$. Unique peptide 1 for neurofilament light polypeptide (NEFL-P1), FTVLTESAAK; unique peptide 1 for neurofilament heavy polypeptide (NEFH-P1), FAQEAEAAR; unique peptide 1 for $\alpha$-internexin (INA-P1), AQLEEASSAR; unique peptide 1 for glial fibrillary acidic protein (GFAP-P1), ALAAELNQLR; unique peptide 1 for microtubule-associated protein 2 (MAP2-P1), GLSSVPEVAEVETTTK; unique peptide 1 for microtubule-associated protein tau (MAPT-P1), TPSLPTPPTR; unique peptide 1 for tubulin beta-3 chain (TUBB3-P1), YLTVATVFR. * $p<0.05$; ** $p<0.01$; *** $p<0.001$.

\subsection{Site-Specificity of Myelin-Related Proteins in Intact and Infarcted Brain Tissues}

Myelin basic protein (MBP), myelin proteolipid protein (PLP) and 2',3'-cyclic-nucleotide 3'-phosphodiesterase (CNP) are major constituents of the CNS myelin [21,22]. Hall et al. found that $39 \%$ of patients with acute ischemic stroke had positive test results for blood MBP on admission [6]. Jauch et al. found that the concentration of MBP increased at $24 \mathrm{~h}$ after treatment [23]. Our results show that MBP, PLP and CNP were present more abundantly in zones 5 and 6 than in zone 4 (Figure 5A, Table S2, $n=12$ ), and also more abundant in zones 2 and 3 than in zone 1 (Figure 5B, Table S2, $n=12$ ). In other words, MBP, PLP and CNP are all site-specific (Figure 5A,B). Therefore, the location of lesions may have to be considered for future clinical studies. 
A

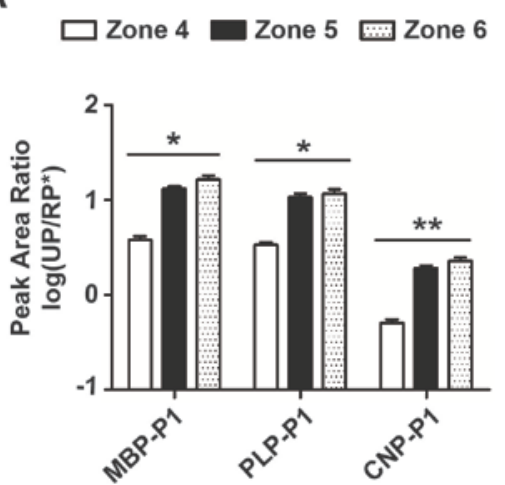

C

$\square$ Zone $4 \square$ Zone 5 Wone 6

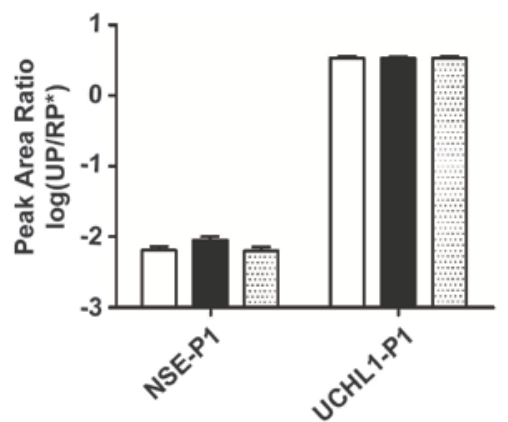

B 曰Zone 1 Zone 2 Z Zone 3

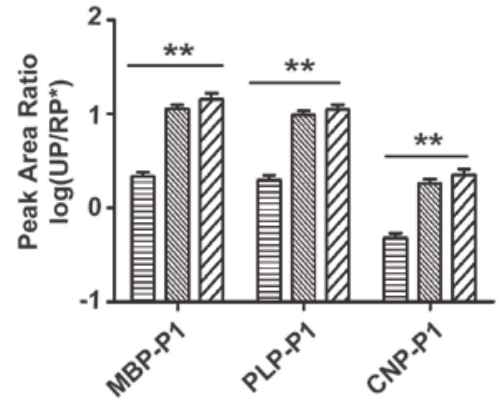

D Zone 1 Zone 2 ZD Zone 3

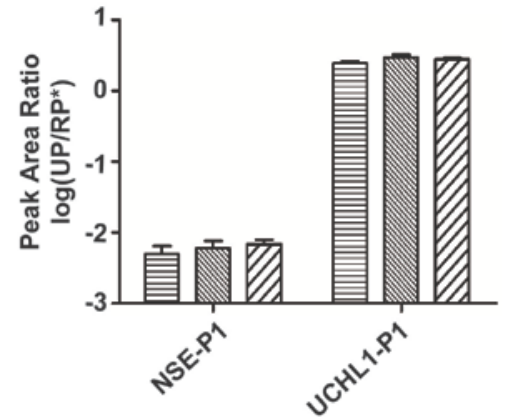

Figure 5. Distribution of myelin proteins and other biomarker candidates in intact and infarcted brain tissue. (A) Three myelin proteins from zones 4-6 of intact brain tissues were verified and semi-quantified by LCM-LRP. Each protein was verified by $2-4$ unique peptides and quantified by the peak area. Peak area ratios of unique peptides to RP* were calculated (UP/RP*) ( $n=12)$; (B) Three unique myelin proteins in zones 1-3 of infarcted brain tissue were collected by LCM, and then the peak area ratios of 3 unique peptides to RP* were calculated (UP/RP*) $(n=12)$; $(\mathbf{C})$ Two protein biomarker candidates from zones 4-6 were verified by 2-3 unique peptides for each and quantified by the peak area. Peak area ratios of unique peptides to RP* were calculated (UP/RP*) $(n=12)$; (D) The peak area ratios of NSE-P1 and UCHL1-P1 (UP/RP*) in zones $1-3$ were calculated $(n=12)$. Unique peptide 1 for myelin basic protein (MBP-P1), DTGILDSIGR; unique peptide 1 for myelin proteolipid protein (PLP-P1), TSASIGSLCADAR; unique peptide 1 for 2',3'-cyclic-nucleotide 3'-phosphodiesterase (CNP-P1), AIFTGYYGK; unique peptide 1 for neuron-specific enolase (NSE-P1), LGAEVYHTLK; unique peptide 1 for ubiquitin carboxyl-terminal hydrolase isozyme L1 (UCHL1-P1), FSAVALCK. * $p<0.05$; ${ }^{* *} p<0.01$.

Neuron-specific enolase (NSE) is a glycolytic enzyme in neurons, also known as gamma-enolase or enolase 2 (ENO2). Ubiquitin carboxyl-terminal hydrolase isozyme L1 (UCHL1) is abundantly present in all neurons [24]. Both NSE and UCHL1 are soluble proteins in the cytoplasm that are not thought to be site-specific, and our results confirm that they are evenly distributed in zones 4-6 and 1-3 (Figure 5C,D). 


\subsection{Abundance and Stability Analysis of Protein Biomarker Candidates by LCM-LRP}

Peak area ratios (UP/RP*) of 12 unique peptides in $5 \mu$ g of brain tissue homogenates were shown to estimate the abundance of protein biomarker candidates (Figure 6A, $n=4$ ). By LCM-LRP analysis, we can also estimate the stability of proteins in the center of infarcted regions by the peak area ratios for zone 1 to zone 4 (UP/UP, 1:4) (Figure 6B, $n=12$ ). Peak area ratios (UP/RP*) of unique peptides of NEFL, INA, GFAP, MAP2, CNP, NSE and UCHL1 in zone 1 were similar to those in zone 4 (Figures 4 and 5, $n=12$ ), and their peak area ratios (UP/UP, 1:4) were close to 1.0 (Figure 6B, $n=12$ ), which suggests that these potential biomarkers are stable in the center of ischemic brain tissue. The peak area ratios (UP/UP, 1:4) of unique peptides of MAPT, TUBB3, MBP and PLP were significantly lower than 1.0 (Figure 6B, $p<0.05$ ), which indicates that these proteins may be degraded at $4 \mathrm{~h}$ after ischemia and are not ideal protein biomarker candidates. The peak area ratio (UP/UP, 1:4) of NEFH unique peptide 1 is $16.275 \pm 6.982$ (Figure $6 \mathrm{~B}, p<0.01, n=12$ ), which suggests that NEFH protein concentration is increased in the early stage of ischemia $(\leq 4 \mathrm{~h})$.

A

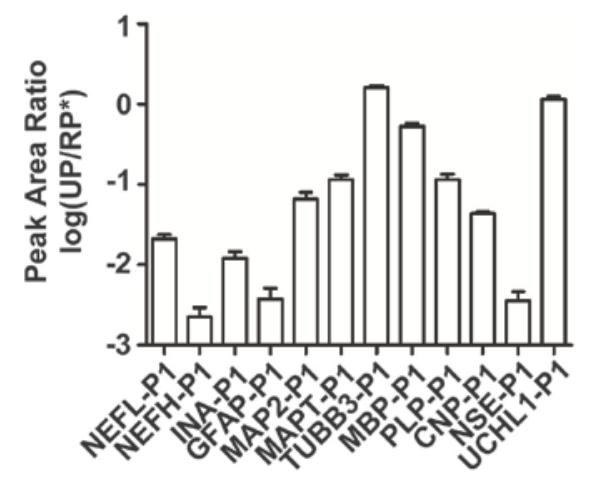

B

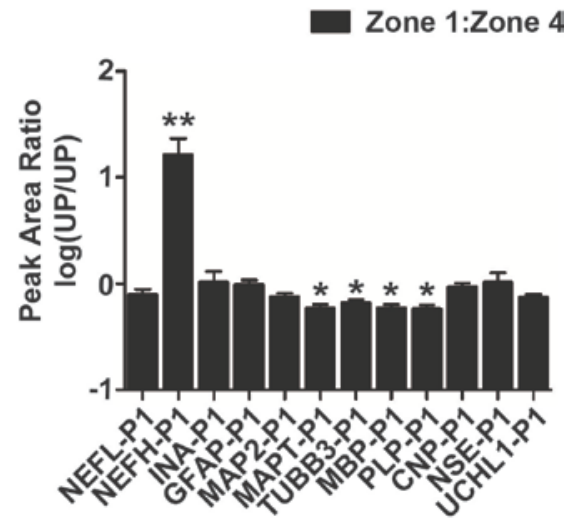

Figure 6. Abundance and stability analysis of protein biomarker candidates by LCM-LRP. (A) Twelve protein biomarker candidates in brain tissue homogenates were verified and semi-quantified by triple-quadrupole mass spectrometry. The abundance of each protein was estimated by the peak area ratio of its corresponding unique peptide ( $n=12)$; (B) Peak areas of 12 unique peptides in zone 1 were compared to those in zone $4(1: 4)$, and the relative peak area ratios (UP/UP) are shown $(n=12){ }^{*} p<0.05 ; * * p<0.01$.

\section{Experimental Section}

\subsection{Materials}

Chloral hydrate was purchased from KeLong Chemical (Chengdu, China). Deionized water was purified by a Millipore Advantage A10 system (Millipore, Bedford, MA, USA). An amount of 90\% ethanol (HPLC grade) was purchased from Thermo Fisher Scientific (Rockford, IL, USA). Solutions of $70 \%(v / v)$ ethanol were prepared with purified $\mathrm{H}_{2} \mathrm{O}$. Xylene was purchased from Sigma Aldrich (St. Louis, MO, USA). Mayer's hematoxylin solution and wosin Y solution were purchased from Merck (Whitehouse Station, NJ, USA). The 2,3,5-triphenytetrazolium chloride (TTC) was obtained from Sigma Aldrich (St. Louis, MO, USA). Optical cutting temperature (OCT) compound was purchased 
from Leica (Wetzlar, Germany). Arcturus CapSure Macro LCM Caps and PEN membrane frame slides were purchased from Life Technologies (Foster City, CA, USA). Laser-capture microdissection apparatus: Arcturus ${ }^{\mathrm{XT}}$ (Life Technologies, Foster City, CA, USA). Cryostat microtome: CM1950 (Leica, Wetzlar, Germany). Ammonium bicarbonate, dithiothreitol (DTT), iodoacetamide (IAA), trifluoroethonal (TFE) and formic acid (FA) were purchased from Sigma Aldrich (St. Louis, MO, USA). Sequencing grade trypsin was obtained from Promega (Madison, WI, USA). Ammonium formate was purchased from Thermo Fisher Scientific (Rockford, IL, USA). HPLC grade acetonitrile $\left(\mathrm{CH}_{3} \mathrm{CN}\right)$ was obtained from Thermo Fisher Scientific (Rockford, IL, USA). Isotopically labeled peptide with 99.8\% purity: APGLTQALNTK $\left({ }^{13} \mathrm{C}_{6},{ }^{15} \mathrm{~N}_{2}\right)$, and unlabeled peptide with the same sequence with 99.0\% purity: APGLTQALNTK, were purchased from GenScript (Nanjing, China).

\subsection{Animals}

The protocol in the study was approved by the Institutional Animal Care and Use Committee of the China Medical University [SYXK(Liao)20150003]. All efforts were made to ensure the animals' welfare and to lessen the number of animals used. Three young adult male Sprague Dawley rats weighing 250-300 g were allowed free access to food and water before all procedures.

\subsection{Intraluminal Middle Cerebral Artery Occlusion (MCAO) Model}

Rats were weighed and placed in an ether jar until they were immobilized $(n=3)$, and anesthetized with $30 \%$ chloral hydrate in normal saline (intraperitoneally), which was supplemented as necessary during the procedure. Body temperature was monitored and maintained within normal limits with a heating pad. A midline neck incision was made and the soft tissue pulled apart. The right common carotid artery (RCCA) was carefully dissected free from the surrounding nerves (without harming the vagal nerve) and a ligature was made using $5-0$ string. The right external carotid artery (RECA) is then separated and a second knot made. Next, the right internal carotid artery (RICA) is isolated and a knot prepared with a 5-0 string. After obtaining good view of the right internal carotid artery (RICA) and the right pterygopalatine artery (RPA), both arteries were clipped, using a microvascular clip. A small hole was cut in the RCCA before it was bifurcated into the RECA and the RICA. A monofilament made of 8.0 nylon (with a diameter of $0.26 \mathrm{~mm}, 40 \mathrm{~mm}$ in length, Beijing Cinontech Co., Ltd., Beijing, China) coated with silicon hardener mixture (with a diameter of $0.36 \pm 0.02 \mathrm{~mm}$ ) is then introduced into the RICA, until it stopped at the clip. The clipped arteries were opened while the filament was inserted into the RICA to occlude the origin of the RMCA in the circle of Willis. The third knot on the RICA was closed to fix the filament in position. The wound was closed with a surgical suture. Animals were put in a heated cage for four hours and neurological deficits were evaluated, and then the animals were sacrificed and brain tissues were collected.

\subsection{Brain Tissue Collection and Preparation}

Four hours after MCA occlusion, rats were anesthetized and perfused with $1 \times$ Phosphate Buffered Saline (PBS) $(n=3)$. Then, animals were sacrificed and brain tissue was collected after $4 \mathrm{~h}$ of ischemia. Slices of fresh brain tissue, $2 \mathrm{~mm}$ in thickness, were stained with $1 \%$ TTC to evaluate the 
size and extent of ischemic infarction in each rat. The rest of the tissue was embedded with OCT compound and frozen at $-80{ }^{\circ} \mathrm{C}$ immediately. Fresh-frozen and OCT compound-embedded brain samples were sectioned $(20 \mu \mathrm{m})$ on a cryostat (Leica, CM1950). Each section was placed onto PEN membrane frame slides (Life Technologies, Foster City, CA, USA), followed by H\&E staining and LCM.

Sectioned tissue slides were immediately fixed in $70 \%$ ethanol for $15 \mathrm{~s}$, placed in deionized water for $30 \mathrm{~s}$ to remove OCT and rehydrate the tissue, placed in Mayer's Hematoxylin for $45 \mathrm{~s}$ to stain nuclei, placed in deionized water for $15 \mathrm{~s}$ to remove excess hematoxylin, placed in Scott's Tap Water for $15 \mathrm{~s}$ to change the hue of hematoxylin, placed in $70 \%$ ethanol for $15 \mathrm{~s}$, and then placed in Eosin $\mathrm{Y}$ for $3 \mathrm{~s}$ to stain cytoplasm. Then, H\&E-stained slides were dehydrated in $95 \%$ ethanol for $30 \mathrm{~s}$ twice, followed by dehydration in $100 \%$ ethanol for 30 s twice. Ethanol was then removed through a 3 min bath in xylene three times.

After the tissue was air-dried, both infarcted and intact areas were microdissected with an Arcturus $^{\mathrm{XT}}$ system. The standard LCM protocol was followed [25]. Briefly, the tissue was cut using an ultraviolet laser with 100\% transmission. Specific areas were collected onto CapSure Macro LCM Cap with the size of $\sim 10 \mathrm{~mm}^{2}$ for each area.

\subsection{On-Film Digestion}

The film was carefully peeled off from the top of each cap and put into an Eppendorf tube. Microdissected cells along with the film were lysed in $50 \mu \mathrm{L}$ of trifluoroethanol (TFE)/50 mM ammonium bicarbonate $(1: 1, v: v)$ and then sonicated in a water bath for $20 \mathrm{~min}$ [26,27]. Samples were reduced with dithiothreitol $(10 \mathrm{mM})$ at $50{ }^{\circ} \mathrm{C}$ for $15 \mathrm{~min}$ followed by alkylation with iodoacetamine $(20 \mathrm{mM})$ in the dark at room temperature for $30 \mathrm{~min}$. The buffer was adjusted to $250 \mu \mathrm{L}$ and tryptic digestion performed at $37{ }^{\circ} \mathrm{C}$ overnight on a shaker. The film was picked out before the samples were dried in a speedvac concentrator (Thermo SAVANT SPD1010, ThermoSavant, Holbrook, NY, USA). The digests were resuspended in $0.1 \%$ formic acid. Each sample was spiked with $0.2 \mathrm{ng} / \mu \mathrm{L}$ APGLTQALNTK* and APGLTQALNTK before liquid chromatography/mass spectrometry (LC-MS/MS) analysis.

\subsection{Selection of Unique Peptides, Reference Peptides and Q1/Q3 Transition}

Unique peptides for eight proteins were selected from the database of PeptideAltas and NIST Libraries of Peptide Tandem Mass Spectra Global Protein Machine. Skyline software (version 2.6, MacCoss Lab, University of Washington, Seattle, WA, USA) was used for the peptide selection. The criteria of unique peptides were as follows: (1) the length of peptides is more than 7 amino acids; (2) peptides containing methionine or tryptophan residues are excluded; (3) in peptide sequences, asparagine followed by glycine or proline is avoided; (4) no N-terminal glutamine residues in peptide sequences are selected; (5) uniqueness of selected peptides are confirmed by searching against Uniprot rat database [28].

The reference peptides (APGLTQALNTK* and APGLTQALNTK) were chosen due to their strong MRM transition signals, suitable elution time and low variability in peak area, which have been verified in another study [12]. 
Three MS1/MS2 transition ion pairs were selected to verify a unique peptide, three of which were applied to confirm a protein. Multiple injections of on-film digests were performed to match relative intensities of target ion pair transition signals with those of MRM transition signals observed previously in ion trap MS/MS spectra obtained from the database. Reproducibility of transition signals between different runs was also examined to eliminate the false positive rates of proteins. Collision energy and declustering potential were calculated based on the following empirical equation, which is recommended by Skyline [29]:

$$
\begin{aligned}
& \text { For doubly-charged peptides, } \mathrm{CE}=0.057 \times \mathrm{m} / \mathrm{z}-4.265 \\
& \text { For triply-charged peptides, } \mathrm{CE}=0.031 \times \mathrm{m} / \mathrm{z}+7.082 \\
& \qquad \mathrm{DP}=0.0729 \times \mathrm{m} / \mathrm{z}+31.117
\end{aligned}
$$

MRM acquisition methods were performed with fragment ion-specific tuned Collision Energy (CE) voltages and scheduled retention time. The dynamic MRM option was used for all data acquisition with a target cycle time of $1 \mathrm{~s}$, with a minimum and maximum dwell time of 17 and $333 \mathrm{~ms}$ (60 maximum concurrent MRMs) and a 300-s MRM detection window for MRM transitions.

\subsection{LC-Multiple Reaction Monitoring (MRM) MS Analysis}

A Shimadzu UFLC XR system (Shimadzu Scientific Instruments, Columbia, MD, USA) was used to directly inject $5 \mu \mathrm{L}$ of digest samples onto a reversed phase analytical column (ACQUITY UPLC $\mathrm{BEH} \mathrm{C18} 1.7 \mu \mathrm{m} 3.0 \times 150 \mathrm{~mm}$ column). The column was mounted in an oven maintaining a temperature of $65{ }^{\circ} \mathrm{C}$. Peptides in each injection were separated using a $0.4 \mathrm{~mL} / \mathrm{min}$ flow rate and a gradient from $3 \%$ to $70 \%$ mobile phase $\mathrm{B}$ over a $30 \mathrm{~min}$ run time. Before each run, three-min equilibration was set up. Mobile phase A consisted of $5 \mathrm{mM}$ ammonium formate in $0.1 \% \mathrm{v} / \mathrm{v}$ formic acid, and mobile phase B consisted of $5 \mathrm{mM}$ ammonium formate in $90 \%$ ACN/0.1\% formic acid. The gradient method was adopted based on previous work as followed (time: \% B): 0.1 min: 9\% B; 3 min: 10\% B; 13 min: 17\% B; 13.5 min: 18\% B; 13.6 min: 21\% B; 16.7 min: 22.5\% B; 19.7 min: 26\% B; 21.7 min: 31\% B; 22.5 min: 38\% B; 23.5 min: 70\% B; 26.9 min: 70\% B; 27 min: 3\% B; 30 min: 3\% B.

MRM measurements were performed by an API 4000 ${ }^{\mathrm{TM}}$ (ESI-QQQ) mass spectrometer (AB Sciex, Foster City, CA, USA). The data was acquired and analyzed by AB Sciex’s Analyst software (version 1.6). The parameter settings during acquisition were as follows: $4500 \mathrm{~V}$ capillary voltage, a curtain gas flow of 65 psi (Ultra-High Purity nitrogen), ion source gas 1 flow at 55 psi, ion source gas 2 flow at 55 psi at a temperature of $550{ }^{\circ} \mathrm{C}$, an MS operating pressure of $3.5 \times 10^{-5}$ Torr, and Q1 and Q3 set to unit resolution (0.7 Full Width at Half Maximum).

\subsection{Data Analysis}

Prism4 (GraphPad Inc., La Jolla, CA, USA) software was used for comparisons across different data sets by ANOVA. "Mean \pm SEM" was used to describe the variability within samples. $p$-values of $<0.05$ were considered significant. 


\section{Conclusions}

In summary, LCM-LRP provides a feasible approach for targeted quantitative analysis of valuable protein samples, and is a very powerful tool of biomarker candidate screening and evaluation. In the present study, we found that protein biomarker candidates, including NEFL, INA, MAP2, MBP, PLP and CNP, are site-specific, but potential protein biomarkers, such as NEFH, GFAP, MAPT, TUBB3, NSE and UCHL1, are evenly distributed in the brain. These findings provide useful information for the design and analysis of relevant clinical studies in the future.

\section{Supplementary Materials}

Supplementary materials can be found at http://www.mdpi.com/1422-0067/16/06/13427/s1.

\section{Acknowledgments}

Wentao Zhang and Catherine Carpenter's significant contribution in the editing process of this manuscript are highly appreciated. The support of the 2013 annual Guangdong key laboratory of enzyme and fermentation under funding number FJ2013001 is gratefully acknowledged.

\section{Author Contributions}

Bing Gao conceived the study, designed and analyzed all experiments. Tingting Lian performed animal surgery and laser captured microdissection. Daixin Qu, Tingting Lian and Xu Zhao performed the mass spectrometry experiments. Lixia Yu performed labeled reference peptide analysis. Bing Gao wrote the paper.

\section{Conflicts of Interest}

The authors declare no conflict of interest.

\section{References}

1. Jickling, G.C.; Sharp, F.R. Blood biomarkers of ischemic stroke. Neurotherapeutics 2011, 8, 349-360.

2. Fernandez-Lopez, D.; Faustino, J.; Daneman, R.; Zhou, L.; Lee, S.Y.; Derugin, N.; Wendland, M.F.; Vexler, Z.S. Blood-brain barrier permeability is increased after acute adult stroke but not neonatal stroke in the rat. J. Neurosci. 2012, 32, 9588-9600.

3. Cui, X.; Chopp, M.; Zacharek, A.; Karasinska, J.M.; Cui, Y.; Ning, R.; Zhang, Y.; Wang, Y.; Chen, J. Deficiency of brain ATP-binding cassette transporter A-1 exacerbates blood-brain barrier and white matter damage after stroke. Stroke 2015, 46, 827-834.

4. Saenger, A.K.; Christenson, R.H. Stroke biomarkers: Progress and challenges for diagnosis, prognosis, differentiation, and treatment. Clin. Chem. 2010, 56, 21-33.

5. Jensen, M.B.; Chacon, M.R.; Sattin, J.A.; Aleu, A.; Lyden, P.D. The promise and potential pitfalls of serum biomarkers for ischemic stroke and transient ischemic attack. Neurologist 2008, 14, 243-246. 
6. Hill, M.D.; Jackowski, G.; Bayer, N.; Lawrence, M.; Jaeschke, R. Biochemical markers in acute ischemic stroke. Can. Med. Assoc. J. 2000, 162, 1139-1140.

7. Emmert-Buck, M.R.; Wulfkuhle, J.D.; Calvert, V.S.; van Meter, A.; Zhou, W.; Coukos, G.; Geho, D.H.; Petricoin, E.F., 3rd.; Liotta, L.A. Laser capture microdissection. Science 1996, 274, 998-1001.

8. Subramanian, M.; Holt, A.G.; Mueller, P.J. Physical activity correlates with glutamate receptor gene expression in spinally-projecting RVLM neurons: A laser capture microdissection study. Brain Res. 2014, 1585, 51-62.

9. Hebbar, S.; Schulz, W.D.; Sauer, U.; Schwudke, D. Laser capture microdissection coupled with on-column extraction LC-MS( $n$ ) enables lipidomics of fluorescently labeled Drosophila neurons. Anal. Chem. 2014, 86, 5345-5352.

10. Bagnato, C.; Thumar, J.; Mayya, V.; Hwang, S.I.; Zebroski, H.; Claffey, K.P.; Haudenschild, C.; Eng, J.K.; Lundgren, D.H.; Han, D.K. Proteomics analysis of human coronary atherosclerotic plaque: A feasibility study of direct tissue proteomics by liquid chromatography and tandem mass spectrometry. Mol. Cell. Proteomics 2007, 6, 1088-1102.

11. Guzel, C.; Ursem, N.T.; Dekker, L.J.; Derkx, P.; Joore, J.; van Dijk, E.; Ligtvoet, G.; Steegers, E.A.; Luider, T.M. Multiple reaction monitoring assay for pre-eclampsia related calcyclin peptides in formalin fixed paraffin embedded placenta. J. Proteome Res. 2011, 10, 3274-3282.

12. Zhang, H.; Liu, Q.; Zimmerman, L.J.; Ham, A.J.; Slebos, R.J.; Rahman, J.; Kikuchi, T.; Massion, P.P.; Carbone, D.P.; Billheimer, D.; et al. Methods for peptide and protein quantitation by liquid chromatography-multiple reaction monitoring mass spectrometry. Mol. Cell. Proteomics 2011, 10, M110 006593.

13. Longa, E.Z.; Weinstein, P.R.; Carlson, S.; Cummins, R. Reversible middle cerebral artery occlusion without craniectomy in rats. Stroke 1989, 20, 84-91.

14. Krueger, M.; Bechmann, I.; Immig, K.; Reichenbach, A.; Härtig, W.; Michalski, D. Blood-brain barrier breakdown involves four distinct stages of vascular damage in various models of experimental focal cerebral ischemia. J. Cereb. Blood Flow Metab. 2014, 35, 292-303.

15. Herrmann, M.; Vos, P.; Wunderlich, M.T.; de Bruijn, C.H.; Lamers, K.J. Release of glial tissue-specific proteins after acute stroke: A comparative analysis of serum concentrations of protein S-100B and glial fibrillary acidic protein. Stroke 2000, 31, 2670-2677.

16. Foerch, C.; Curdt, I.; Yan, B.; Dvorak, F.; Hermans, M.; Berkefeld, J.; Raabe, A.; Neumann-Haefelin, T.; Steinmetz, H.; Sitzer, M. Serum glial fibrillary acidic protein as a biomarker for intracerebral haemorrhage in patients with acute stroke. J. Neurol. Neurosurg. Psychiatry 2006, 77, 181-184.

17. Laser-Azogui, A.; Kornreich, M.; Malka-Gibor, E.; Beck, R. Neurofilament assembly and function during neuronal development. Curr. Opin. Cell Biol. 2015, 32C, 92-101.

18. Liu, Y.; Staal, J.A.; Canty, A.J.; Kirkcaldie, M.T.; King, A.E.; Bibari, O.; Mitew, S.T.; Dickson, T.C.; Vickers, J.C. Cytoskeletal changes during development and aging in the cortex of neurofilament light protein knockout mice. J. Comp. Neurol. 2013, 521, 1817-1827.

19. Delacourte, A.; Defossez, A. Alzheimer's disease: Tau proteins, the promoting factors of microtubule assembly, are major components of paired helical filaments. J. Neurol. Sci. 1986, 76, 173-186. 
20. Yushmanov, V.E.; Kharlamov, A.; Simplaceanu, E.; Williams, D.S.; Jones, S.C. Differences between arterial occlusive and cortical photothrombosis stroke models with magnetic resonance imaging and microtubule-associated protein-2 immunoreactivity. Magn. Reson. Imaging 2006, 24, 1087-1093.

21. Trotter, J.L.; Clark, H.B.; Collins, K.G.; Wegeschiede, C.L.; Scarpellini, J.D. Myelin proteolipid protein induces demyelinating disease in mice. J. Neurol. Sci. 1987, 79, 173-188.

22. Sheedlo, H.J.; Sprinkle, T.J. The distribution of 2':3'-cyclic nucleotide 3'-phosphodiesterase (CNP) in the CNS of normal (+/+) and Shiverer (shi/shi) mice. J. Neurol. Sci. 1985, 67, 35-44.

23. Jauch, E.C.; Lindsell, C.; Broderick, J.; Fagan, S.C.; Tilley, B.C.; Levine, S.R.; NINDS rt-PA Stroke Study Group. Association of serial biochemical markers with acute ischemic stroke: The National Institute of Neurological Disorders and Stroke recombinant tissue plasminogen activator Stroke Study. Stroke 2006, 37, 2508-2513.

24. Osaka, H.; Wang, Y.L.; Takada, K.; Takizawa, S.; Setsuie, R.; Li, H.; Sato, Y.; Nishikawa, K.; Sun, Y.J.; Sakurai, M.; et al. Ubiquitin carboxy-terminal hydrolase L1 binds to and stabilizes monoubiquitin in neuron. Hum. Mol. Genet. 2003, 12, 1945-1958.

25. Erickson, H.S.; Albert, P.S.; Gillespie, J.W.; Rodriguez-Canales, J.; Marston Linehan, W.; Pinto, P.A.; Chuaqui, R.F.; Emmert-Buck, M.R. Quantitative RT-PCR gene expression analysis of laser microdissected tissue samples. Nat. Protoc. 2009, 4, 902-922.

26. Wang, H.; Qian, W.J.; Mottaz, H.M.; Clauss, T.R.; Anderson, D.J.; Moore, R.J.; Camp, D.G., 2nd.; Khan, A.H.; Sforza, D.M.; Pallavicini, M.; et al. Development and evaluation of a micro- and nano-scale proteomic sample preparation method. J. Proteome Res. 2005, 4, 2397-2403.

27. Sprung, R.W., Jr.; Brock, J.W.; Tanksley, J.P.; Li, M.; Washington, M.K.; Slebos, R.J.; Liebler, D.C. Equivalence of protein inventories obtained from formalin-fixed paraffin-embedded and frozen tissue in multidimensional liquid chromatography-tandem mass spectrometry shotgun proteomic analysis. Mol. Cell. Proteomics 2009, 8, 1988-1998.

28. Lange, V.; Picotti, P.; Domon, B.; Aebersold, R. Selected reaction monitoring for quantitative proteomics: A tutorial. Mol. Syst. Biol. 2008, 4, 222.

29. MacLean, B.; Tomazela, D.M.; Shulman, N.; Chambers, M.; Finney, G.L.; Frewen, B.; Kern, R.; Tabb, D.L.; Liebler, D.C.; MacCoss, M.J. Skyline: An open source document editor for creating and analyzing targeted proteomics experiments. Bioinformatics 2010, 26, 966-968.

(C) 2015 by the authors; licensee MDPI, Basel, Switzerland. This article is an open access article distributed under the terms and conditions of the Creative Commons Attribution license (http://creativecommons.org/licenses/by/4.0/). 